\title{
Positive rational interpolatory quadrature formulas on the unit circle and the interval
}

\author{
Karl Deckers ${ }^{\mathrm{a}, 1,2, *}$, Adhemar Bultheel ${ }^{\mathrm{a}, 2}$, Ruymán Cruz-Barroso ${ }^{\mathrm{b}, 3}$, Francisco Perdomo-Pío $^{\mathrm{b}, 3,4}$ \\ ${ }^{a}$ Department of Computer Science, K.U.Leuven, Celestijnenlaan 200 A, B-3001 Heverlee, Belgium. \\ ${ }^{b}$ Department of Mathematical Analysis, La Laguna University, 38271 La Laguna, Tenerife. Canary Islands. Spain.
}

\begin{abstract}
We present a relation between rational Gauss-type quadrature formulas that approximate integrals of the form $J_{\mu}(F)=\int_{-1}^{1} F(x) d \mu(x)$, and rational Szegó quadrature formulas that approximate integrals of the form $I_{\dot{\mu}}(F)=\int_{-\pi}^{\pi} F\left(e^{\mathbf{i} \theta}\right) d \stackrel{\circ}{\mu}(\theta)$. The measures $\mu$ and $\stackrel{\circ}{\mu}$ are assumed to be positive bounded Borel measures on the interval $[-1,1]$ and the complex unit circle respectively, and are related by $\stackrel{\circ}{\mu}^{\prime}(\theta)=\mu^{\prime}(\cos \theta)|\sin \theta|$. Next, making use of the so-called para-orthogonal rational functions, we obtain a one-parameter family of rational interpolatory quadrature formulas with positive weights for $J_{\mu}(F)$. Finally, we include some illustrative numerical examples.
\end{abstract}

Keywords: Positive rational interpolatory quadrature, rational Szegő quadrature, rational Gauss-type quadratures, para-orthogonal rational functions, interval, unit circle 2000 MSC: 42C05, 65D32

\section{Introduction}

In this paper we shall explore the interplay between quadrature formulas for integrals of the form $J_{\mu}(F)=\int_{-1}^{1} F(x) d \mu(x)$, where $\mu$ is a positive Borel measure on $I=[-1,1]$, and integrals of the form $I_{\dot{\mu}}(F)=\int_{-\pi}^{\pi} F\left(e^{\mathbf{i} \theta}\right) d \stackrel{\circ}{\mu}(\theta)$, where $\stackrel{\circ}{\mu}$ is supported on $[-\pi, \pi]$. The integral $I_{\dot{\mu}}$ can also be considered as an integral over the unit circle $\mathbb{T}$ of the complex plane, which we shall currently do to refer to the two different cases. The Joukowski transform is a well known two-to-one mapping relating the interval $I$ and the complex unit circle $\mathbb{T}$, which can be most easily characterized by

\section{${ }^{*}$ Corresponding author}

Email address: Karl. Deckers@cs . kuleuven. be (Karl Deckers)

${ }^{1}$ This author is a Postdoctoral Fellow of the Research Foundation - Flanders (FWO).

${ }^{2}$ The work of these authors is partially supported by the Fund of Scientific Research (FWO), project "RAM: Rational modelling: optimal conditioning and stable algorithms”, grant $\sharp$ G.0423.05 and the Belgian Network DYSCO (Dynamical Systems, Control, and Optimization), funded by the Interuniversity Attraction Poles Programme, initiated by the Belgian State, Science Policy Office. The scientific responsibility rests with the authors.

${ }^{3}$ The work is partially supported by Dirección General de Programas y Transferencia de Conocimiento, Ministerio de Ciencia e Innovación of Spain under grant MTM 2008-06671.

${ }^{4}$ The work of the fourth author has been partially supported by a Grant of Agencia Canaria de Investigación, Innovación y Sociedad de la Información del Gobierno de Canarias. 
the relations $z=e^{\mathbf{i} \theta} \in \mathbb{T} \Leftrightarrow x=\cos \theta \in I$. It is used for example in Szegô's book [19] to relate orthogonal polynomials on $I$ with orthogonal polynomials on $\mathbb{T}$ whenever the measures are related by $\stackrel{\circ}{\mu}^{\prime}(\theta)=\mu^{\prime}(\cos \theta)|\sin \theta|$. The corresponding quadrature formulas can be related as well. For example in [4], Gaussian quadrature formulas on I correspond to Szegô quadrature formulas on $\mathbb{T}$. Both of them have distinct nodes in the support of the measure, the weights are positive and they have a maximal domain of validity in the space of polynomials on $I$ and the space of Laurent polynomials on $\mathbb{T}$ respectively.

The classical Gauss-Radau and Gauss-Lobatto quadrature formulas correspond to prefixing one and two nodes, respectively, in I, while Szegő-Radau and Szegó-Lobatto quadrature formulas correspond to prefixing one and two nodes, respectively, on $\mathbb{T}$. The remaining nodes are freely chosen inside $I$, respectively $\mathbb{T}$, to achieve the maximal domain of validity while maintaining positive weights. This was elaborated e.g. in [3] for $I$ and $[2,15]$ for $\mathbb{T}$.

For integrals with an integrand having singularities close to $I$, respectively $\mathbb{T}$, it may be advantageous to consider also poles for the space where the quadrature is exact. To obtain these spaces of rational functions, one has to extend the theory of orthogonal polynomials to the theory of orthogonal rational functions with prescribed poles; see [6]. The poles are assumed to be outside the support $I$, and hence, their image is outside $\mathbb{T}$. The relation between the orthogonal functions on $I$ and $\mathbb{T}$ has been described in [25] in the case of real poles and more generally in [11]. In this paper we shall consider poles that are arbitrary complex (possibly at infinity), except for the last pole in the sequence of poles (which is assumed real or at infinity), but still outside $I$, hence with Joukowski images outside $\mathbb{T}$. This relation then allows us to give precise relations that are essentially one-to-one between rational Gaussian quadrature formulas and rational Szegő quadrature formulas. Also the case of Radau and Lobatto, with one and two prescribed nodes, respectively, will be considered for the rational case as they were in $[3,7,8,15]$. Also here, precise connections between the two classes of quadrature formulas will be shown.

The paper is organized as follows: In Section 2 we introduce the necessary notation, and recall the definitions and properties needed in Section 3, which contains the main results. There, the exact relation between nodes and weights of the rational Gaussian, Gauss-Radau, and Gauss-Lobatto for the interval, and the nodes and weights of the rational Szegő, Szegő-Radau, and Szegő-Lobatto quadrature formulas will be proved. Finally, in Section 4 we illustrate the formulas with some numerical examples.

\section{Preliminaries}

The field of complex numbers will be denoted by $\mathbb{C}$ and the Riemann sphere by $\overline{\mathbb{C}}=\mathbb{C} \cup\{\infty\}$. For the real line we use the symbol $\mathbb{R}$ and for the extended real line $\overline{\mathbb{R}}=\mathbb{R} \cup\{\infty\}$. Let $a \in \mathbb{C}$, then $\mathfrak{R}\{a\}$ refers to the real part of $a$, while $\mathfrak{I}\{a\}$ refers to the imaginary part. Further, we denote the imaginary unit by $\mathbf{i}$. The unit circle and the open unit disk are denoted respectively by $\mathbb{T}=\{z \in$ $\mathbb{C}:|z|=1\}$ and $\mathbb{D}=\{z \in \mathbb{C}:|z|<1\}$. Whenever the value zero is omitted in a set $X \subseteq \overline{\mathbb{C}}$, this will be represented by $X_{0}$. Similarly, the complement of a set $Y \subset \overline{\mathbb{C}}$ with respect to a set $X \subseteq \overline{\mathbb{C}}$ will be given by $X_{Y}$; i.e., $X_{Y}=\{t \in X: t \notin Y\}$. Further, if $b=\lceil a\rceil$ with $a \in \mathbb{R}$, then $b$ is the smallest integer so that $b \geq a$. If, on the other hand, $b=\lfloor a\rfloor$ with $a \in \mathbb{R}$, then $b$ is the largest integer so that $b \leq a$. 
In this paper, we will consider quadrature formulas on the unit circle $\mathbb{T}$ and on the interval $I=[-1,1]$. Although $z$ and $x$ are both complex variables, we reserve the notation $z$ for the unit circle and $x$ for the interval.

For any complex function $f(t)$, with $t=z$ or $t=x$, we define the involution operation or substar conjugate by $f_{*}(t)=\overline{f(1 / \bar{t})}$. Next, we define the super-c conjugate by $f^{c}(t)=\overline{f(\bar{t})}$, and consequently $f_{*}^{c}$ by $f_{*}^{c}(t)=f(1 / t)$. Note that, if $f(t)$ has a pole at $t=p$, then $f_{*}(t)$ (respectively $f^{c}(t)$ and $\left.f_{*}^{c}(t)\right)$ has a pole at $t=1 / \bar{p}$ (respectively $t=\bar{p}$ and $t=1 / p$ ). Further, with $f^{\text {inv }}$ we denote the inverse of the function $f$, to avoid confusion with the notation $f^{-1}=1 / f$.

Let a sequence of complex poles $\mathcal{A}_{n}=\left\{\alpha_{1}, \alpha_{2}, \ldots, \alpha_{n}\right\} \subset \overline{\mathbb{C}}_{I}$ be fixed. Unless stated otherwise, we will assume that $\alpha_{n} \in \overline{\mathbb{R}}_{I}$ while the poles in $\mathcal{A}_{n-1}$ are arbitrary complex or infinite; hence, they do not have to appear in pairs of complex conjugates. Define the basis functions

$$
b_{0}(x) \equiv 1, \quad b_{k}(x)=b_{k-1}(x) Z_{k}(x), \quad \text { with } \quad Z_{k}(x)=\frac{x}{1-x / \alpha_{k}}, \quad k=1,2, \ldots, n .
$$

These basis functions generate the nested spaces of rational functions with poles in $\mathcal{A}_{n}$ defined by

$$
\mathcal{L}_{j}=\mathcal{L}\left\{\alpha_{1}, \ldots, \alpha_{j}\right\}=\operatorname{span}\left\{b_{0}, \ldots, b_{j}\right\}=\left\{p_{j} / \pi_{j}: p_{j} \in \mathcal{P}_{j}\right\}, 0 \leq j \leq n,
$$

where $\mathcal{P}_{n}$ denotes the space of polynomials of degree less than or equal to $n$, and

$$
\pi_{0}(x) \equiv 1, \quad \pi_{j}(x)=\prod_{k=1}^{j}\left(1-x / \alpha_{k}\right), 0<j \leq n .
$$

With the definition of the super-c conjugate we define $\mathcal{L}_{j}^{c}=\left\{f: f^{c} \in \mathcal{L}_{j}\right\}$. Note that $\mathcal{L}_{j}$ and $\mathcal{L}_{j}^{c}$ are rational generalizations of $\mathcal{P}_{j}$. Indeed, if all $\alpha_{k}=\infty$, the expressions in (1) become $Z_{k}(x)=$ $Z_{k}^{c}(x)=x$ and $b_{k}(x)=b_{k}^{c}(x)=x^{k}$.

Consider the integral

$$
J_{\mu}(F)=\int_{-1}^{1} F(x) d \mu(x)
$$

where $\mu$ is a positive bounded Borel measure on $I$. To approximate $J_{\mu}(F)$, where $F$ is a function with singularities (possibly close to, but) outside the interval, rational interpolatory quadrature formulas are often preferred. An $n$th rational interpolatory quadrature is obtained by integrating an interpolating rational function of degree $n-1$ (at the most), and is of the form

$$
J_{n}(F)=\sum_{k=1}^{n} \lambda_{k} F\left(x_{k}\right),\left\{x_{k}\right\}_{k=1}^{n} \subset I, x_{j} \neq x_{k} \text { if } j \neq k,\left\{\lambda_{k}\right\}_{k=1}^{n} \subset \mathbb{R},
$$

so that $J_{\mu}(F)=J_{n}(F)$ for every $F \in \mathcal{R}_{p, q}=\mathcal{L}_{p} \cdot \mathcal{L}_{q}^{c}=\left\{f \cdot g^{c}: f \in \mathcal{L}_{p}, g \in \mathcal{L}_{q}\right\}$, with $p+q \leq 2 n-1$ and $0 \leq q \leq p \leq n$. The space $\mathcal{R}_{p, q}$ is then called the domain of validity.

\footnotetext{
${ }^{5}$ The degree of a rational function is defined as the maximum of the degree of the polynomials in the numerator and denominator.
} 
The weights $\lambda_{k}$ in the quadrature formula are real iff

$$
\mathcal{R}_{p, q}=\mathcal{R}_{p, q}^{c}
$$

Indeed, suppose the weights are real. Then, for any $F \in \mathcal{R}_{p, q}^{c}$ we have that

$$
J_{\mu}(F)=\overline{J_{\mu}\left(F^{c}\right)}=\overline{J_{n}\left(F^{c}\right)}=\sum_{k=1}^{n} \overline{\lambda_{k} F^{c}\left(x_{k}\right)}=\sum_{k=1}^{n} \lambda_{k} F\left(x_{k}\right),
$$

which means that the quadrature is exact for any $F \in \mathcal{R}_{p, q}^{c}$ as well. Vice versa, suppose $\mathcal{R}_{p, q}=\mathcal{R}_{p, q}^{c}$. Then, for any $F \in \mathcal{R}_{p, q}$ we have that

$$
\sum_{k=1}^{n} \lambda_{k} F\left(x_{k}\right)=J_{\mu}(F)=\overline{J_{\mu}\left(F^{c}\right)}=\sum_{k=1}^{n} \bar{\lambda}_{k} F\left(x_{k}\right) .
$$

From here it is easy to check that the weights are all real. Note that for $p=q=n-1$, the equality in (2) holds true for every sequence of poles, and that the equality also holds true for $p=n$ and $q=n-1$ due to the earlier assumption that $\alpha_{n} \in \overline{\mathbb{R}}_{I}$.

Let $\varphi_{n} \in \mathcal{L}_{n} \backslash \mathcal{L}_{n-1}$ denote an $n$th orthogonal rational function (ORF) with respect to the inner product

$$
\langle F, G\rangle_{\mu}=\int_{-1}^{1} F(x) G^{c}(x) d \mu(x) .
$$

The zeros $x_{k}$ of $\varphi_{n}(x)$ are all distinct and in the open interval $(-1,1)$, and hence, can be chosen as nodes for the quadrature formula $J_{n}(F)$. In this way, we obtain the $n$-point rational Gaussian quadrature formula, which has maximal domain of validity; i.e., the approximation is exact for every function $F \in \mathcal{R}_{n, n-1}$. It is well known that the weights in the rational Gaussian quadrature are all positive. See also [6, Chapt. 11.6], [21], [13, Thm. 2.3], and [9, Thm. 2.3.5]).

For any other choice of nodes, the weights may be non-positive and the quadrature will only be exact in a smaller set of rational functions. For each node that is fixed in advance, the domain of validity will generally ${ }^{6}$ decrease by one. It is reasonable though to require that we have at least exactness for $p+q=n-1$. A special case is obtained when one node in the $n$-point quadrature is fixed in advance, so that the weights are all positive and the quadrature is exact for every $F \in \mathcal{R}_{n-1, n-1}$, which corresponds to the $n$-point rational Gauss-Radau quadrature formula. However, the existence of this $n$-point rational Gauss-Radau quadrature depends on the choice of the node (e.g., it surely does not exist whenever the node is a zero of $\varphi_{n-1}$; see [10]). Whenever two nodes in an $(n+1)$-point quadrature formula are fixed in advance, so that the weights are all positive and the quadrature is exact for every $F \in \mathcal{R}_{n, n-1}$, we obtain the $(n+1)$-point rational Gauss-Lobatto quadrature formula.

\footnotetext{
${ }^{6}$ For some specific choices for the nodes, the domain of validity may remain the same or may even decrease more (see also the recent paper [3] for the polynomial case).
} 
Another sequence of basis functions will be used for the unit circle. Given a sequence of complex numbers $\mathcal{B}_{n}=\left\{\beta_{1}, \beta_{2}, \ldots, \beta_{n}\right\} \subset \mathbb{D}$, with $\beta_{n} \in(-1,1)$, we define the Blaschke factors for $\mathcal{B}_{n}$ as

$$
\zeta_{k}(z)=\eta_{k} \frac{z-\beta_{k}}{1-\bar{\beta}_{k} z}, \quad \eta_{k}=\left\{\begin{array}{cc}
-\frac{\bar{\beta}_{k}}{\left|\beta_{k}\right|}, & \beta_{k} \neq 0 \\
1, & \beta_{k}=0
\end{array}, k=1,2, \ldots, n,\right.
$$

and the corresponding Blaschke products for $\mathcal{B}_{n}$ as

$$
B_{0}(z) \equiv 1, \quad B_{k}(z)=B_{k-1}(z) \zeta_{k}(z), k=1,2, \ldots, n .
$$

These Blaschke products generate the nested spaces of rational functions $\stackrel{\circ}{\mathcal{L}}_{j}=\operatorname{span}\left\{B_{0}, \ldots, B_{j}\right\}$, $0 \leq j \leq n$. Define

$$
\stackrel{\circ}{\pi}_{0}(z) \equiv 1, \quad \stackrel{\circ}{\pi}_{j}(z)=\prod_{k=1}^{j}\left(1-\bar{\beta}_{k} z\right), 0<j \leq n,
$$

then we may write equivalently

$$
B_{j}(z)=v_{j} \frac{\stackrel{\circ}{j}_{j}^{*}(z)}{\dot{\pi}_{j}(z)}, \quad v_{j}=\prod_{k=1}^{j} \eta_{k} \in \mathbb{T},
$$

where $\stackrel{\circ}{\pi}_{j}^{*}(z)=z^{j} \stackrel{\circ}{\pi}_{j *}(z)$, and $\stackrel{\circ}{\mathcal{L}}_{j}=\left\{p_{j} / \stackrel{\circ}{\pi}_{j}: p_{j} \in \mathcal{P}_{j}\right\}$.

With the definition of the substar conjugate and the super-c conjugate we can define $\mathcal{L}_{j *}=$ $\left\{f: f_{*} \in \dot{\circ}_{j}\right\}, \stackrel{\circ}{\mathcal{L}}_{j}^{c}=\left\{f: f^{c} \in \dot{\circ}_{j}\right\}$ and $\dot{\circ}_{j *}^{c}=\left\{f: f_{*}^{c} \in \stackrel{\circ}{\mathcal{L}}_{j}\right\}$. Note that $\dot{\circ}_{j}$ and $\dot{\mathcal{L}}_{j}^{c}$ are rational generalizations of $\mathcal{P}_{j}$ too. Indeed, if all $\beta_{k}=0$ (or equivalently, $1 / \bar{\beta}_{k}=\infty$ for every $k$ ), the expression in (3) becomes $\zeta_{k}(z)=\zeta_{k}^{c}(z)=z$ and the expression in (4) becomes $B_{k}(z)=B_{k}^{c}(z)=z^{k}$.

Consider now the integral

$$
I_{\dot{\mu}}(F)=\int_{-\pi}^{\pi} F(z) d \stackrel{\circ}{\mu}(\theta), z=e^{\mathbf{i} \theta}
$$

where $\stackrel{\circ}{\mu}$ is a positive bounded Borel measure on $\mathbb{T}^{8}$ The rational interpolatory quadrature formulas to approximate $I_{\dot{\mu}}(F)$ are then of the form

$$
I_{n}(F)=\sum_{k=1}^{n} \stackrel{\lambda}{\lambda}_{k} F\left(z_{k}\right),\left\{z_{k}=e^{\mathbf{i} \theta_{k}}\right\}_{k=1}^{n} \subset \mathbb{T}, z_{j} \neq z_{k} \text { if } j \neq k,\left\{\stackrel{\lambda}{k}_{k}\right\}_{k=1}^{n} \subset \mathbb{R},
$$

so that $I_{\dot{\mu}}(F)=I_{n}(F)$ for every $F \in \stackrel{\circ}{\mathcal{R}}_{p, q}=\stackrel{\circ}{\mathcal{L}}_{p} \cdot \stackrel{\circ}{\mathcal{L}}_{q^{*}}$, with $n-1 \leq p+q \leq 2 n-2$ and $0 \leq q \leq p \leq n-1$. Clearly, the weights are real now iff $\stackrel{\circ}{R}, q=\stackrel{\circ}{\mathcal{R}}_{(p, q) *}$, implying that $p=q$.

\footnotetext{
${ }^{7}$ The factors and products are named after Wilhelm Blaschke, who introduced these for the first time in [1].

${ }^{8}$ The measure $\stackrel{\circ}{\mu}$ on $\mathbb{T}$ induces a measure on $[-\pi, \pi]$ for which we shall use the same notation $\stackrel{\circ}{\mu}$.
} 
Let $\phi_{n} \in \stackrel{\circ}{\mathcal{L}}_{n} \backslash \stackrel{\circ}{\mathcal{L}}_{n-1}$ denote an $n$th ORF with respect to the inner product

$$
\langle F, G\rangle_{\dot{\mu}}=\int_{-\pi}^{\pi} F(z) G_{*}(z) d \stackrel{\circ}{\mu}(\theta) .
$$

The leading coefficient $\stackrel{\circ}{\kappa}_{n}$ (i.e., the coefficient of $B_{n}(z)$ in the expansion of $\phi_{n}(z)$ in the basis $\left.\left\{B_{0}, \ldots, B_{n}\right\}\right)$ is then given by $\stackrel{\circ}{k}_{n}=\overline{\phi_{n}^{*}\left(\beta_{n}\right)}$, where $\phi_{n}^{*}(z)=B_{n}(z) \phi_{n *}(z)$. In the remainder we will assume the ORFs $\phi_{n}$ are monic; i.e., $\stackrel{\circ}{k}_{n}=1$. We then define a para-orthogonal rational function (pORF)

$$
\stackrel{\circ}{Q}, \tau(z)=\phi_{n}(z)+\tau \phi_{n}^{*}(z), \tau \in \mathbb{T} .
$$

The zeros $z_{k}$ of $\stackrel{Q}{n, \tau}_{n}(z)$ are all distinct and on the unit circle $\mathbb{T}$, and hence, can be chosen as nodes for the quadrature formula $I_{n}(F)$. This way, we obtain an $n$-point rational Szegô quadrature formula, which has maximal domain of validity $(p=q=n-1)$. Again, it is well known that in this case the weights $\stackrel{\circ}{\lambda}_{k}$ are all positive.

Due to the presence of the parameter $\tau$ in (6), the nodes and weights in an $n$-point rational Szegó quadrature formula are (unlike in the case of the interval) not unique. For the same reason, an $n$-point rational Szegő-Radau quadrature formula (one fixed node) always has positive weights and maximal domain of validity too. While an $n$-point rational Szegő-Lobatto quadrature formula (two fixed nodes) is at least exact for every $F \in \stackrel{\circ}{\mathcal{R}}_{n-2, n-2}$ and again always has positive weights (see e.g. [7, 8]).

We denote the Joukowski Transformation $x=\frac{1}{2}\left(z+z^{-1}\right)$ by $x=J(z)$, mapping the open unit disc $\mathbb{D}$ onto the cut Riemann sphere $\overline{\mathbb{C}}_{I}$ and the unit circle $\mathbb{T}$ onto the interval $I$. When $z=e^{\mathbf{i} \theta}$, then $x=J(z)=\cos \theta$. In this paper we will assume that $x$ and $z$ are related by this transformation. The inverse mapping is denoted by $z=J^{i n v}(x)$ and is chosen so that $z \in \mathbb{D}$ if $x \in \overline{\mathbb{C}}_{I}$. With the sequence $\mathcal{A}_{n}=\left\{\alpha_{1}, \alpha_{2}, \ldots, \alpha_{n}\right\} \subset \overline{\mathbb{C}}_{I}$ we associate a sequence $\mathcal{B}_{n}=\left\{\beta_{1}, \beta_{2}, \ldots, \beta_{n}\right\} \subset \mathbb{D}$, so that $\beta_{k}=J^{i n v}\left(\alpha_{k}\right)$, and $\hat{\mathcal{B}}_{2 n}=\left\{\hat{\beta}_{1}, \ldots, \hat{\beta}_{2 n}\right\} \subset \mathbb{D}$ with

$$
\hat{\beta}_{2 k}=\overline{\hat{\beta}}_{2 k-1}=\beta_{k}, \quad k=1, \ldots, n .
$$

In the remainder we will consistently use the hat in our notation to refer to the sequence of numbers (7).

A connection between quadrature formulas on the unit circle and the interval $I$ is given in e.g. [4] and [5]. If $\mu$ is a measure on $I$, we obtain a measure on $\mathbb{T}$ by setting

$$
\stackrel{\circ}{\mu}(E)=\mu(\{\cos \theta, \theta \in E \cap[0, \pi)\})+\mu(\{\cos \theta, \theta \in E \cap[-\pi, 0)\}),
$$

which can also be written as $\stackrel{\mu}{\mu}(E)=\int_{E}|d \mu(\cos \theta)|$. Using the Lebesgue decomposition of $\mu$ and the change-of-variables theorem (see e.g. [18, p. 153]) it is not difficult to see that $\mu^{\prime}(\theta)=$ $\mu^{\prime}(\cos \theta)|\sin \theta|$ (see also [19]). Note that by the Joukowski Transform, a function $F(x)$ transforms into a function $\stackrel{\circ}{F}(z)=(F \circ J)(z)$, so that $\stackrel{\circ}{F}(z)=\stackrel{\circ}{F}\left(z^{-1}\right)$ and $J_{\mu}(F)=\frac{1}{2} I_{\dot{\mu}}(\stackrel{\circ}{F})$. Moreover, from [12, Lem. 3.1] it follows that every function $F \in \mathcal{L}_{k} \backslash \mathcal{L}_{k-1}$ transforms into a function $\stackrel{\circ}{F} \in\left(\stackrel{\circ}{\mathcal{L}}_{k}^{c} \cdot \stackrel{\circ}{\mathcal{L}}_{k *}\right) \backslash\left(\stackrel{\mathscr{L}}{c}_{k-1}^{c} \cdot \stackrel{\circ}{\mathcal{L}}_{(k-1) *}\right)$; see also [9, Chapt. 3.2]. 


\section{Positive rational interpolatory quadrature formulas}

In [4] it is shown that in the polynomial case there is an intimate relationship between the Gauss-type (i.e., Gaussian, Gauss-Radau and Gauss-Lobatto) and certain Szegő quadrature formulas whenever the measures $\mu$ and $\stackrel{\circ}{\mu}$ are related by (8). In this respect, the Szegô quadrature formulas are based on the zeros of para-orthogonal polynomials with $\tau \in\{ \pm 1\}$. Later, making use of the para-orthogonal polynomials with $\tau \neq \pm 1$, the same authors have obtained a one-parameter family of interpolatory quadrature formulas with positive weights for $J_{\mu}(F)$ in [5]. The aim of this section is to generalize these results to the case of rational functions. First, we need the following lemma concerning the zeros of an $n$th pORF and the weights in an $n$-point rational Szegó quadrature formula that is based on the zeros of the pORF $\stackrel{\circ}{n, \pm 1}_{(}(z)=\phi_{n}(z) \pm \phi_{n}^{*}(z)$.

Lemma 3.1. Suppose the numbers $\mathcal{B}_{n-1}=\left\{\beta_{1}, \ldots, \beta_{n-1}\right\}$ are real or appear in complex conjugate pairs. Assume $\stackrel{\circ}{\mu}$ is defined as before by $(8)$, and let ${\stackrel{\circ}{Q}, \tau_{n}}_{n}(z)=\phi_{n}(z)+\tau_{n} \phi_{n}^{*}(z)$ be an nth pORF with respect to $\stackrel{\circ}{\mu}$ with $\tau_{n} \in \mathbb{T}$. Then,

1. The zeros of ${\stackrel{Q}{n, \tau_{n}}}_{(z)}$ appear in complex conjugate pairs iff $\tau_{n}= \pm 1$.

2. ${\stackrel{Q}{n, \tau_{n}}}_{2}(z)$ has a zero in

(a) $z=1$ iff $\tau_{n}=-v_{n}$,

(b) $z=-1$ iff $\tau_{n}=(-1)^{n+1} v_{n}$,

where $v_{n} \in\{ \pm 1\}$ is defined as before in (5).

3. Let $I_{n}(F)=\sum_{k=1}^{n} \grave{\lambda}_{k} F\left(z_{k}\right)$ be an n-point rational Szegó quadrature formula for $I_{\dot{\mu}}(F)=$ $\int_{-\pi}^{\pi} F(z) d \mu(\theta)$, based on the zeros of the $p O R F \stackrel{\circ}{Q}_{n, \pm 1}(z)$. Then for $k=1, \ldots, n$, the weight $\stackrel{\lambda}{k}_{k}$ corresponding to the node $z_{k}$ is equal to the weight $\lambda_{j}$ corresponding to the node $z_{j}=\bar{z}_{k}$.

Proof. Suppose $\xi$ and $\bar{\xi}$ are zeros of $\grave{Q}_{n, \tau_{n}}(z)$. Then $\phi_{n}(\xi)+\tau_{n} \phi_{n}^{*}(\xi)=0$ and $\phi_{n}(\bar{\xi})+\tau_{n} \phi_{n}^{*}(\bar{\xi})=0$. Thus,

$$
0=\overline{\phi_{n}(\bar{\xi})+\tau_{n} \phi_{n}^{*}(\bar{\xi})}=\phi_{n}^{c}(\xi)+\overline{\tau_{n}} \phi_{n}^{c *}(\xi),
$$

and since $\phi_{n}^{c}(z)=\phi_{n}(z)$ it holds that

$$
0=\phi_{n}^{c}(\xi)+\bar{\tau}_{n} \phi_{n}^{c *}(\xi)=\phi_{n}(\xi)+\bar{\tau}_{n} \phi_{n}^{*}(\xi) .
$$

Consequently, $\tau_{n}=-\phi_{n}(\xi) / \phi_{n}^{*}(\xi)=\bar{\tau}_{n}$, which ends the proof of the first part.

Let now $\xi \in\{ \pm 1\}$, then we have that ${\stackrel{\circ}{n, \tau_{n}}}(\xi)=0$ iff

$$
0=\phi_{n}(\xi)+\tau_{n} \phi_{n}^{*}(\xi)=\phi_{n}(\xi)+\tau_{n} B_{n}(\xi) \overline{\phi_{n}(\xi)}=\phi_{n}(\xi)+\tau_{n} B_{n}(\xi) \phi_{n}^{c}(\xi)
$$

Since $\phi_{n}^{c}(z)=\phi_{n}(z)$ and $B_{n}(\xi)=v_{n} \xi^{n}$, it holds that

$$
0=\phi_{n}(\xi)+\tau_{n} B_{n}(\xi) \phi_{n}^{c}(\xi)=\left(1+\tau_{n} v_{n} \xi^{n}\right) \phi_{n}(\xi) .
$$

Because the zeros of $\phi_{n}(z)$ are all in $\mathbb{D}$ (see [6, Cor. 3.2.2(3)]), it follows that $\phi_{n}(\xi) \neq 0$. As a result, ${\stackrel{\circ}{Q}, \tau_{n}}(\xi)=0$ iff $\tau_{n}=-v_{n} \xi^{n}$. This concludes the proof of the second part. 
Finally, note that for the given sequence of numbers $\mathcal{B}_{n-1}$ it holds that $\stackrel{\circ}{\mathcal{R}}_{(n-1, n-1) *}^{c}=\stackrel{\circ}{\mathcal{R}}_{n-1, n-1}$. Consequently, because the measure $\stackrel{\mu}{\mu}$ is symmetric, we have for every $F \in \stackrel{\circ}{\mathcal{R}}_{n-1, n-1}$ that

$$
\sum_{k=1}^{n} \grave{\lambda}_{k} F\left(z_{k}\right)=I_{\dot{\mu}}(F)=I_{\dot{\mu}}\left(F_{*}^{c}\right)=\sum_{k=1}^{n} \grave{\lambda}_{k} F_{*}^{c}\left(z_{k}\right)=\sum_{k=1}^{n} \stackrel{\curlywedge}{\lambda}_{k} F\left(\bar{z}_{k}\right) .
$$

Since for every index $k$ for which $\bar{z}_{k} \neq z_{k}$, there exists an index $j \neq k$ so that $\bar{z}_{k}=z_{j}$, the proof is concluded.

Let $\varphi_{n} \in \mathcal{L}_{n} \backslash \mathcal{L}_{n-1}$ represent an $n$th ORF with respect to the measure $\mu$ on $I$, and $\phi_{n} \in \dot{\mathcal{L}}_{n} \backslash \dot{\mathcal{L}}_{n-1}$ the $n$th monic ORF with respect to the measure $\stackrel{\mu}{\mu}$ on $\mathbb{T}$, given by (8). In [11, Thm. 5] $]^{9}$ it has been proved that there exists a nonzero constant $C_{n}$ so that

$$
\varphi_{n}(x)=C_{n} B_{n *}(z)\left\{\hat{\phi}_{2 n}^{c}(z)+\hat{\phi}_{2 n}^{*}(z)\right\}=C_{n} B_{n *}(z) \hat{Q}_{2 n, 1}(z) .
$$

The second equality here holds true because the numbers in this sequence are real or appear in complex conjugate pairs, and the last pole $\hat{\beta}_{2 n}=\beta_{n}$ is real, so that $\hat{\phi}_{2 n}^{c}(z)=\hat{\phi}_{2 n}(z)$. The relation given by (9) is a rational generalization of the connection between orthogonal polynomials (OPs) with respect to the measure $\stackrel{\circ}{ }$ on the unit circle and OPs with respect to the measure $\mu$ on the interval, which has been established by Szegó in his famous book [19, Thm. 11.5], and played a crucial role in connecting Gaussian with Szegó quadrature formulas. Consequently, with this rational generalization we can prove the following two theorems.

Theorem 3.2. Let $\stackrel{\circ}{\mu}$ be given by (8), and let $\hat{I}_{2 n}(F)=\sum_{k=1}^{2 n} \stackrel{\leftrightarrow}{\lambda}_{k} F\left(z_{k}\right)$ be a 2 n-point rational Szegö quadrature formula for $I_{\dot{\mu}}(F)=\int_{-\pi}^{\pi} F(z) d \mu(\theta)$, based on the zeros of the $p O R F \hat{Q}_{2 n, 1}(z)=\hat{\phi}_{2 n}(z)+$ $\hat{\phi}_{2 n}^{*}(z)$. Suppose the zeros are ordered in such a way that $z_{n+k}=\bar{z}_{k}$ for $k=1, \ldots, n$, with $z_{k} \neq \bar{z}_{j}$ for every $1 \leq k<j \leq n$, and set $z_{k}=e^{\mathbf{i} \theta_{k}}$ for $k=1, \ldots, n$. Then, when taking $x_{k}=\cos \theta_{k}$ and $\lambda_{k}=\grave{\lambda}_{k}$ for $k=1, \ldots, n$, the formula $J_{n}(F)=\sum_{k=1}^{n} \lambda_{k} F\left(x_{k}\right)$ coincides with the n-point rational Gaussian quadrature formula for $J_{\mu}(F)=\int_{-1}^{1} F(x) d \mu(x)$, based on the zeros of an $n$th $\operatorname{ORF} \varphi_{n} \in \mathcal{L}_{n} \backslash \mathcal{L}_{n-1}$.

Proof. From the first and second parts of Lemma 3.1 it follows that $\hat{Q}_{2 n, 1}(z)$ has $2 n$ zeros, all different from 1 and -1 (because $\hat{v}_{2 n}=1$ ), appearing in complex conjugate pairs. Next, from the third part of Lemma 3.1 it then follows that $\dot{\lambda}_{n+k}=\dot{\lambda}_{k}$. Consider now an arbitrary function $F \in \mathcal{R}_{n, n-1}=\mathcal{L}_{n} \cdot \mathcal{L}_{n-1}^{c}=\mathcal{L}_{n}^{c} \cdot \mathcal{L}_{n-1}$. Clearly, its corresponding function $\stackrel{\circ}{F}$ is then in $\left(\stackrel{\circ}{\mathcal{L}}_{n} \cdot \stackrel{\circ}{\mathcal{L}}_{n-1}^{c}\right)^{c}$. $\left(\stackrel{\circ}{\mathcal{L}}_{n} \cdot \stackrel{\circ}{\mathcal{L}}_{n-1}^{c}\right)_{*}=\left(\stackrel{\circ}{\mathcal{L}}_{n} \cdot \dot{\mathscr{L}}_{n-1}^{c}\right) \cdot\left(\stackrel{\circ}{\mathcal{L}}_{n} \cdot \stackrel{\circ}{\mathcal{L}}_{n-1}^{c}\right)_{*}=\stackrel{\circ}{\mathcal{R}}_{2 n-1,2 n-1}$. Consequently,

$$
\begin{aligned}
J_{n}(F) & =\sum_{k=1}^{n} \lambda_{k} F\left(x_{k}\right)=\sum_{k=1}^{n} \stackrel{\circ}{\lambda}_{k} \stackrel{\circ}{F}\left(z_{k}\right)=\frac{1}{2} \sum_{k=1}^{2 n} \stackrel{\circ}{\lambda}_{k} \stackrel{\circ}{F}\left(z_{k}\right) \\
& =\frac{1}{2} \hat{I}_{2 n}(\stackrel{\circ}{F})=\frac{1}{2} I_{\dot{\mu}}(\stackrel{\circ}{F})=J_{\mu}(F) .
\end{aligned}
$$

\footnotetext{
${ }^{9}$ In [11] the measure $\mu$ was assumed to be absolutely continuous, but this can easily be extended to arbitrary positive Borel measures whose support is an infinite set. See also [14, p. 190] for the polynomial case.
} 
Finally, because the equality holds for every $F \in \mathcal{R}_{n, n-1}$, the $n$-point formula is the $n$-point rational Gaussian quadrature formula.

Conversely we have the following theorem.

Theorem 3.3. Let $J_{n}(F)=\sum_{k=1}^{n} \lambda_{k} F\left(x_{k}\right)$ be the n-point rational Gaussian quadrature formula for $J_{\mu}(F)=\int_{-1}^{1} F(x) d \mu(x)$, based on the zeros of an nth ORF $\varphi_{n} \in \mathcal{L}_{n} \backslash \mathcal{L}_{n-1}$. Set $x_{k}=\cos \theta_{k}$, and define $\left\{z_{k}\right\}_{k=1}^{2 n}$ and $\left\{\stackrel{1}{\lambda}_{k}\right\}_{k=1}^{2 n}$ by means of

$$
\left.\begin{array}{ll}
z_{k}=e^{\mathbf{i} \theta_{k}}, & \stackrel{\circ}{\lambda}_{k}=\lambda_{k} \\
z_{n+k}=e^{-\mathbf{i} \theta_{k}}, & \stackrel{\lambda}{n+k}_{n+\lambda}=\lambda_{k}
\end{array}\right\}, k=1, \ldots, n .
$$

Further, let $\stackrel{\mu}{\mu}$ be given by (8). Then $\hat{I}_{2 n}(F)=\sum_{k=1}^{2 n} \stackrel{\leftrightarrow}{\lambda}_{k} F\left(z_{k}\right)=\sum_{k=1}^{n} \stackrel{\circ}{\lambda}_{k}\left[F\left(z_{k}\right)+F\left(\bar{z}_{k}\right)\right]$ coincides with a 2 -point rational Szegó quadrature formula for $I_{\hat{\mu}}(F)=\int_{-\pi}^{\pi} F(z) d \stackrel{\circ}{\mu}(\theta)$, with nodes the zeros of the $p O R F \hat{Q}_{2 n, 1}(z)=\hat{\phi}_{2 n}(z)+\hat{\phi}_{2 n}^{*}(z)$.

Proof. Consider an arbitrary function $F \in \mathcal{R}_{n, n-1}$ and its corresponding function $\stackrel{\circ}{F} \in \stackrel{\circ}{\mathcal{R}}_{2 n-1,2 n-1}$. We then have that

$$
\begin{aligned}
\hat{I}_{2 n}(\stackrel{\circ}{F}) & =\sum_{k=1}^{2 n} \stackrel{\circ}{\lambda}_{k} \stackrel{\circ}{F}\left(z_{k}\right)=2 \sum_{k=1}^{n} \stackrel{\circ}{\lambda}_{k} \stackrel{\circ}{F}\left(z_{k}\right)=2 \sum_{k=1}^{n} \lambda_{k} F\left(x_{k}\right) \\
& =2 J_{n}(F)=2 J_{\mu}(F)=I_{\dot{\mu}}(\stackrel{\circ}{F}) .
\end{aligned}
$$

Hence, the $2 n$-point rational formula is exact for every function $\stackrel{\circ}{F} \in \stackrel{\circ}{\mathcal{R}}_{2 n-1,2 n-1}$ for which $\stackrel{\circ}{F}(z)=$ $\stackrel{\circ}{F}\left(z^{-1}\right)$. Consider now the pORF $\stackrel{\hat{Q}}{2 n, 1}(z)=\hat{\phi}_{2 n}(z)+\hat{\phi}_{2 n}^{*}(z)$ with zeros in $\left\{\tilde{z}_{k}\right\}_{k=1}^{2 n}$. From the first and second parts of Lemma 3.1 it follows that $\hat{Q}_{2 n, 1}(z)$ has $2 n$ zeros, all different from 1 and -1 (because $\hat{v}_{2 n}=1$ ), appearing in complex conjugate pairs. Thus, suppose they are ordered in such a way that $\tilde{z}_{k}=\overline{\tilde{z}}_{n+k}=e^{\mathbf{i} \tilde{\boldsymbol{\theta}}_{k}}$ for $k=1, \ldots, n$, and consider the $2 n$-point rational Szegó quadrature formula $\tilde{I}_{2 n}(F)=\sum_{k=1}^{2 n} \tilde{\grave{\lambda}}_{k} F\left(\tilde{z}_{k}\right)$ for $I_{\dot{\mu}}(F)=\int_{-\pi}^{\pi} F(z) d \mu(\theta)$. From the third part of Lemma 3.1 it then follows that $\tilde{\grave{\lambda}}_{n+k}=\tilde{\grave{\lambda}}_{k}$. So, let us again consider an arbitrary function $F \in \mathcal{R}_{n, n-1}$ and its corresponding function $\stackrel{\circ}{F} \in \stackrel{\circ}{\mathcal{R}}_{2 n-1,2 n-1}$. Since the $2 n$-point rational Szegó quadrature formula $\tilde{I}_{2 n}(\stackrel{\circ}{F})$ is exact for every $\stackrel{\circ}{F} \in \stackrel{\circ}{\mathcal{R}}_{2 n-1,2 n-1}$, it follows from the previous theorem that the $n$-point formula $\tilde{J}_{n}(F)=\sum_{k=1}^{n} \tilde{\lambda}_{k} F\left(\tilde{x}_{k}\right)$, with $\tilde{x}_{k}=\cos \tilde{\theta}_{k}$ and $\tilde{\lambda}_{k}=\tilde{\grave{\lambda}}_{k}$, is an $n$-point rational Gaussian quadrature formula too. Because the orthonormal rational functions $\varphi_{j}, j=1, \ldots, n$, are (up to a unimodular factor) unique, and hence, the nodes and weights in the $n$-point rational Gaussian quadrature formula are unique too (see the proof of [9, Thm. 2.3.5]), it follows that $J_{n}(F)$ coincides with $\tilde{J}_{n}(F)$, and hence, that $\hat{I}_{2 n}(F)$ coincides with $\tilde{I}_{2 n}(F)$.

Next, consider the complex varying measure $\mu_{n-1}$ defined by (see $[9$, p. 200])

$$
d \mu_{n-1}(x)=\frac{1}{2}\left(\beta_{n-1}-\frac{1}{\bar{\beta}_{n-1}}\right) \sqrt{\alpha_{n}^{2}-1} \frac{\left(1-x^{2}\right)}{\left(\bar{\alpha}_{n-1}-x\right)\left|\alpha_{n}-x\right|} d \mu(x),
$$


and let $\tilde{\varphi}_{n-1}(x)$ denote an $(n-1)$ th ORF on $I$ with respect to this measure $\mu_{n-1}$, associated with the sequence $\mathcal{A}_{n-1} \subset \overline{\mathbb{C}}_{I}$. Further, let $\phi_{n} \in \stackrel{\circ}{\mathcal{L}}_{n} \backslash \stackrel{\circ}{\mathcal{L}}_{n-1}$ denote the $n$th monic ORF with respect to the measure $\stackrel{\circ}{\mu}$ on $\mathbb{T}$, given by (8). From [11, Thm. 6] it then follows that there exists a nonzero constant $C_{n-1}$ so that

$$
\begin{aligned}
\tilde{\varphi}_{n-1}(x) & =C_{n-1} \frac{B_{n *}(z)}{\zeta_{n}^{c}(z)-\zeta_{n *}(z)}\left\{\hat{\phi}_{2 n}^{c}(z)-\hat{\phi}_{2 n}^{*}(z)\right\} \\
& =\tilde{C}_{n-1}\left(1-\beta_{n} z\right)^{2} B_{(n-1) *}(z)\left\{\frac{\hat{\grave{Q}}_{2 n,-1}(z)}{z^{2}-1}\right\}, \tilde{C}_{n-1}=\frac{C_{n-1}}{1-\beta_{n}^{2}}
\end{aligned}
$$

where $\zeta_{n}(z)$ is defined as before in (3). Again, the second equality holds true because the numbers in this sequence are real or appear in complex conjugate pairs, and the last pole $\hat{\beta}_{2 n}=\beta_{n}$ is real, so that $\hat{\phi}_{2 n}^{c}(z)=\hat{\phi}_{2 n}(z)$ and $\zeta_{n}^{c}(z)=\zeta_{n}(z)=\zeta_{n *}^{-1}(z)$. The relation given by (10) is now a rational generalization of the connection between OPs with respect to the measure $\stackrel{\mu}{\mu}$ on the unit circle and OPs with respect to the measure $\tilde{\mu}$, with $d \tilde{\mu}(x)=\left(1-x^{2}\right) d \mu(x)$, on the interval, which has been established by Szegó in his book [19, p. 294], and played a crucial role in connecting GaussLobatto (with fixed nodes in 1 and -1) with Szegô quadrature formulas. Consequently, with this rational generalization we can now prove the following two theorems whose proofs are analogs to those for Theorems 3.2 and 3.3.

Theorem 3.4. Let $\stackrel{\circ}{\mu}$ be given by (8), and let $\hat{I}_{2 n}(F)=2 A F(-1)+2 B F(1)+\sum_{k=1}^{2 n-2} \stackrel{\circ}{\lambda}_{k} F\left(z_{k}\right)$ be a 2n-point rational Szegó quadrature formula for $I_{\mu}(F)=\int_{-\pi}^{\pi} F(z) d \mu(\theta)$, based on the zeros of the pORF $\hat{\grave{Q}}_{2 n,-1}(z)=\hat{\phi}_{2 n}(z)-\hat{\phi}_{2 n}^{*}(z)$. Suppose the zeros are ordered in such a way that $z_{n-1+k}=\bar{z}_{k}$ for $k=1, \ldots, n-1$, with $z_{k} \neq \bar{z}_{j}$ for every $1 \leq k<j \leq n-1$, and set $z_{k}=e^{\mathbf{i} \theta_{k}}$ for $k=$ $1, \ldots, n-1$. Then, when taking $x_{k}=\cos \theta_{k}$ and $\lambda_{k}=\lambda_{k}$ for $k=1, \ldots, n-1$, the formula $J_{n+1}(F)=A F(-1)+B F(1)+\sum_{k=1}^{n-1} \lambda_{k} F\left(x_{k}\right)$ coincides with the $(n+1)$-point rational Gauss-Lobatto quadrature formula for $J_{\mu}(F)=\int_{-1}^{1} F(x) d \mu(x)$ with fixed nodes in 1 and -1 .

Conversely we have the following theorem.

Theorem 3.5. Let $J_{n+1}(F)=A F(-1)+B F(1)+\sum_{k=1}^{n-1} \lambda_{k} F\left(x_{k}\right)$ be the $(n+1)$-point rational GaussLobatto quadrature formula for $J_{\mu}(F)=\int_{-1}^{1} F(x) d \mu(x)$ with fixed nodes in 1 and -1 . Set $x_{k}=\cos \theta_{k}$ and define $\left\{z_{k}\right\}_{k=1}^{2 n-2}$ and $\left\{\stackrel{\circ}{k}_{k}\right\}_{k=1}^{2 n-2}$ by means of

$$
\left.\begin{array}{ll}
z_{k}=e^{\mathbf{i} \theta_{k}}, & \stackrel{\circ}{k}_{k}=\lambda_{k} \\
z_{n-1+k}=e^{-\mathbf{i} \theta_{k}}, & \stackrel{\leftrightarrow}{n}_{n-1+k}=\lambda_{k}
\end{array}\right\}, k=1, \ldots, n-1 .
$$

Further, let $\stackrel{\rho}{\mu}$ be given by (8). Then the formula $\hat{I}_{2 n}(F)=2 A F(-1)+2 B F(1)+\sum_{k=1}^{2 n-2} \stackrel{\lambda}{k}_{k} F\left(z_{k}\right)=$ $2 A F(-1)+2 B F(1)+\sum_{k=1}^{n-1} \dot{\lambda}_{k}\left[F\left(z_{j}\right)+F\left(\bar{z}_{j}\right)\right]$ coincides with a $2 n$-point rational Szegó quadrature formula for $I_{\dot{\mu}}(F)=\int_{-\pi}^{\pi} F(z) d \stackrel{\circ}{\mu}(\theta)$, with nodes the zeros of the pORF $\hat{Q}_{2 n,-1}(z)=\hat{\phi}_{2 n}(z)-\hat{\phi}_{2 n}^{*}(z)$. 
Like above, where we have connected rational Gaussian and Gauss-Lobatto with rational Szegô quadrature formulas, let us see now what happens in the case of rational Gauss-Radau quadrature formulas with a fixed node at either 1 or -1 . For this, we will need the following theorem.

Theorem 3.6. Let $\phi_{n}$ denote the monic ORF with respect to the measure $\stackrel{\circ}{\mu}$ on $\mathbb{T}$, given by (8), and define $\breve{\varphi}_{n-1}^{ \pm} \in \mathcal{L}_{n-1}$ by

$$
\begin{aligned}
\breve{\varphi}_{n-1}^{ \pm}(x) & =\frac{B_{(n-1) *}(z)}{1 \pm \eta_{n} \zeta_{n}(z)}\left\{\hat{\phi}_{2 n-1}(z) \pm \eta_{n} \hat{\phi}_{2 n-1}^{*}(z)\right\} \\
& =\frac{B_{(n-1) *}(z)}{1 \pm \eta_{n} \zeta_{n}(z)} \hat{Q}_{2 n-1, \pm \eta_{n}}(z),
\end{aligned}
$$

where $\hat{\beta}_{2 n-1}=\bar{\beta}_{n}=\beta_{n}$. Then it holds that

$$
Q_{n}^{ \pm}(x)=\left(\frac{1 \pm x}{1-x / \alpha_{n}}\right) \breve{\varphi}_{n-1}^{ \pm}(x)
$$

is orthogonal to $\mathcal{L}_{n-1}\left(\alpha_{n}\right)=\left\{\frac{\left(1-x / \alpha_{n}\right) p_{n-2}(x)}{\pi_{n-1}(x)}: p_{n-2} \in \mathcal{P}_{n-2}\right\}$ with respect to the measure $\mu$ on $I$.

Proof. First, note that $\hat{v}_{2 n-1}=\eta_{n} \in\{ \pm 1\}$, so that $\hat{\stackrel{Q}{Q}}_{2 n-1, \pm \eta_{n}}(z)$ has a zero in $z=\mp 1$. Next, let us denote the right-hand side of (11) by $R_{n-1}^{ \pm}(z)$. Then with

$$
1 \pm \eta_{n} \zeta_{n}(z)=\frac{(1 \pm z)\left(1 \mp \beta_{n}\right)}{\left(1-\beta_{n} z\right)}
$$

it follows that $R_{n-1}^{ \pm} \in \stackrel{\circ}{\mathcal{L}}_{n-1}^{c} \stackrel{\circ}{\mathcal{L}}_{(n-1) *}$. Moreover, we have that

$$
\begin{aligned}
R_{n-1}^{ \pm}\left(z^{-1}\right) & =\left[R_{n-1}^{ \pm}\right]_{*}^{c}(z) \\
& =\frac{B_{n-1}^{c}(z)}{1 \pm \eta_{n} \zeta_{n *}(z)}\left\{\hat{\phi}_{(2 n-1) *}(z) \pm \eta_{n} B_{n *}(z) B_{(n-1) *}^{c}(z) \hat{\phi}_{2 n-1}(z)\right\} \\
& =\frac{\zeta_{n}(z)}{\zeta_{n}(z) \pm \eta_{n}}\left\{B_{n-1}^{c}(z) \hat{\phi}_{(2 n-1) *}(z) \pm \eta_{n} B_{n *}(z) \hat{\phi}_{2 n-1}(z)\right\} \\
& =\frac{B_{(n-1) *}(z)}{\zeta_{n}(z) \pm \eta_{n}}\left\{\hat{\phi}_{2 n-1}^{*}(z) \pm \eta_{n} \hat{\phi}_{2 n-1}(z)\right\}=R_{n-1}^{ \pm}(z) .
\end{aligned}
$$

Thus, we have proved that $\breve{\varphi}_{n-1}^{ \pm} \in \mathcal{L}_{n-1}$.

Next, with $x=J(z)$ we find after some computations that

$$
\frac{1 \pm x}{1-x / \alpha_{n}}=\frac{\left(1+\beta_{n}^{2}\right)}{2\left(1 \mp \beta_{n}\right)^{2}}\left[1 \pm \eta_{n} \zeta_{n}(z)\right]\left[1 \pm \eta_{n} \zeta_{n *}(z)\right] .
$$

So, setting $C_{n}=\frac{\left(1+\beta_{n}^{2}\right)}{2\left(1+\beta_{n}\right)^{2}}$, we find that

$$
\begin{aligned}
Q_{n}^{ \pm}(x) & =C_{n} B_{(n-1) *}(z)\left\{\hat{\phi}_{2 n-1}(z) \pm \eta_{n} \hat{\phi}_{2 n-1}^{*}(z)\right\}\left[1 \pm \eta_{n} \zeta_{n *}(z)\right] \\
& =C_{n}\left\{\left[1 \pm \eta_{n} \zeta_{n *}(z)\right] \hat{\sigma}_{2 n-1}(z)+\left[1 \pm \eta_{n} \zeta_{n}(z)\right] \hat{\sigma}_{(2 n-1) *}^{c}(z)\right\},
\end{aligned}
$$


where

$$
\hat{\sigma}_{2 n-1}(z)=B_{(n-1) *}(z) \hat{\phi}_{2 n-1}(z)
$$

Consider now an arbitrary function $f \in \mathcal{L}_{n-1}\left(\alpha_{n}\right)$ and its corresponding function $\stackrel{\circ}{f} \in \dot{\mathcal{L}}_{n-1}^{c}$. $\stackrel{\circ}{\mathcal{L}}_{(n-1) *}$. Clearly, this corresponding function $\stackrel{\circ}{f}$ is then of the form

$$
\stackrel{\circ}{f}(z)=\frac{\left(z-\beta_{n}\right) q_{n-2}(z)\left(1-\beta_{n} z\right) q_{n-2}^{*}(z)}{\stackrel{\circ}{\pi}_{n-1}(z) \stackrel{\circ}{\pi}_{n-1}^{*}(z)}, q_{n-2} \in \mathcal{P}_{n-2} .
$$

Furthermore, because $\stackrel{\circ}{f}(z)=\stackrel{\circ}{f}_{*}^{c}(z)$ and the measure $\stackrel{\circ}{\mu}$ is symmetric (i.e., $\forall F: I_{\mu}\left(F_{*}^{c}\right)=I_{\dot{\mu}}(F)$ ), we have that

$$
\left\langle Q_{n}^{ \pm}, f\right\rangle_{\mu}=C_{n}\left\langle\hat{\sigma}_{2 n-1},\left[1 \pm \eta_{n} \zeta_{n}\right] \stackrel{\circ}{f}\right\rangle_{\dot{\mu}}=0,
$$

where the last equality follows from the fact that $\left[1 \pm \eta_{n} \zeta_{n}(z)\right] \stackrel{\circ}{f}(z) \in \stackrel{\circ}{\mathcal{L}}_{n-1}^{c} \cdot \dot{\circ}_{(n-1) *}$, while $\hat{\sigma}_{2 n-1}(z)$ is orthogonal to $\dot{\mathcal{L}}_{n-1}^{c} \cdot \dot{\mathcal{L}}_{(n-1) *}$ (see [11, Thm. 2]). This concludes the proof.

Since $Q_{n}^{ \pm}(x)$ is orthogonal to $\mathcal{L}_{n-1}\left(\alpha_{n}\right)$, this means that $Q_{n}^{ \pm}(x)$ is a quasi-orthogonal rational function (see [6, Chapt. 11.5]) of the form ${ }^{10}$

$$
Q_{n}^{ \pm}(x)=c_{n}\left\{\varphi_{n}(x)+\rho^{ \pm} \frac{Z_{n}(x)}{Z_{n-1}^{c}(x)} \varphi_{n-1}(x)\right\}, c_{n} \in \mathbb{C}_{0},
$$

where $\varphi_{k}(x), k=n-1, n$, denotes a $k$ th ORF with respect to the measure $\mu$ on $I$, and

$$
\rho^{ \pm}=-\frac{\varphi_{n}( \pm 1)}{Z_{n}( \pm 1)} \cdot \frac{Z_{n-1}^{c}( \pm 1)}{\varphi_{n-1}( \pm 1)}
$$

Further, note that whenever all the poles are at infinity, the space $\mathcal{L}_{n-1}\left(\alpha_{n}\right)=\mathcal{P}_{n-2}$, so that the previous theorem again is a rational generalization of the connection between OPs with respect to the measure $\stackrel{\circ}{\mu}$ on the unit circle and OPs with respect to the measure $\mu^{ \pm}$, where $d \mu^{ \pm}(x)=$ $(1 \pm x) d \mu(x)$, on the interval (see [4, Thm. 4.4]). Consequently, we can now prove the following two theorems whose proofs are again similar to those for Theorems 3.2 and 3.3.

Theorem 3.7. Suppose $\xi \in\{ \pm 1\}$. Let $\stackrel{\rho}{\mu}$ be given by $(8)$, and let $\hat{I}_{2 n}(F)=2 A F(\xi)+\sum_{k=1}^{2 n-2} \grave{\lambda}_{k} F\left(z_{k}\right)$ be a $(2 n-1)$-point rational Szegó quadrature formula for $I_{\dot{\mu}}(F)=\int_{-\pi}^{\pi} F(z) d \mu(\theta)$, based on the zeros of the pORF $\hat{\grave{Q}}_{2 n-1,-\xi \eta_{n}}(z)=\hat{\phi}_{2 n-1}(z)-\xi \eta_{n} \hat{\phi}_{2 n-1}^{*}(z)$. Suppose the zeros are ordered in such a way that $z_{n-1+k}=\bar{z}_{k}$ for $k=1, \ldots, n-1$, with $z_{k} \neq \bar{z}_{j}$ for every $1 \leq k<j \leq n-1$, and set $z_{k}=e^{\mathbf{i} \theta_{k}}$ for $k=1, \ldots, n-1$. Then, when taking $x_{k}=\cos \theta_{k}$ and $\lambda_{k}=\grave{\lambda}_{k}$ for $k=1, \ldots, n$, the formula $J_{n}(F)=$ $A F(\xi)+\sum_{k=1}^{n-1} \lambda_{k} F\left(x_{k}\right)$ coincides with the n-point rational Gauss-Radau quadrature formula for $J_{\mu}(F)=\int_{-1}^{1} F(x) d \mu(x)$ with fixed node in $\xi$.

\footnotetext{
${ }^{10} \operatorname{In}(12)$ we used the super-c conjugate of $Z_{n-1}(x)$ in the definition of the quasi-orthogonal rational function, because $\alpha_{n-1}$ can be arbitrarily complex. Whereas in [6, Chapt. 11.5], this super-c conjugate does not appear in the definition, because the poles are assumed to be real.
} 
Conversely we have the following theorem.

Theorem 3.8. Suppose $\xi \in\{ \pm 1\}$. Let $J_{n}(F)=A F(\xi)+\sum_{k=1}^{n-1} \lambda_{k} F\left(x_{k}\right)$ be the n-point rational GaussRadau quadrature formula for $J_{\mu}(F)=\int_{-1}^{1} F(x) d \mu(x)$ with fixed node in $\xi$. Set $x_{k}=\cos \theta_{k}$ and define $\left\{z_{k}\right\}_{k=1}^{2 n-2}$ and $\left\{\dot{\lambda}_{k}\right\}_{k=1}^{2 n-2}$ by means of

$$
\left.\begin{array}{ll}
z_{k}=e^{\mathbf{i} \theta_{k}}, & \stackrel{\circ}{\lambda}_{k}=\lambda_{k} \\
z_{n-1+k}=e^{-\mathbf{i} \theta_{k}}, & \stackrel{\lambda}{n-1+k}_{n k}=\lambda_{k}
\end{array}\right\}, k=1, \ldots, n-1 .
$$

Further, let $\stackrel{\circ}{\mu}$ be given by (8). Then $\hat{I}_{2 n-1}(F)=2 A F(\xi)+\sum_{k=1}^{2 n-2} \stackrel{\circ}{\lambda}_{k} F\left(z_{k}\right)=2 A F(\xi)+\sum_{k=1}^{n-1} \stackrel{\leftrightarrow}{\lambda}_{k}\left[F\left(z_{k}\right)+\right.$ $F\left(\bar{z}_{k}\right)$ ] coincides with a (2n-1)-point rational Szegó quadrature formula for $I_{\dot{\mu}}(F)=\int_{-\pi}^{\pi} F(z) d \stackrel{\circ}{\mu}(\theta)$, with nodes the zeros of the pORF $\hat{Q}_{2 n-1,-\xi \eta_{n}}(z)=\hat{\phi}_{2 n-1}(z)-\xi \eta_{n} \hat{\phi}_{2 n-1}^{*}(z)$.

Finally, in the following theorem we give a rational generalization of Theorem 2.2 in [5]; i.e., we prove for $\tau \neq \pm 1$ that, under the condition that $\mathcal{L}_{n-1}=\mathcal{L}_{n-1}^{c}$, the $n$-point rational Szegó quadrature formulas transform into $n$-point rational interpolatory quadrature formulas on the interval with positive weights, which are only exact in $\mathcal{L}_{n-1}$ unless some particular choice of $\tau$ is made.

Theorem 3.9. Suppose the poles $\left\{\alpha_{1}, \ldots, \alpha_{n-1}\right\}$ (and hence, the numbers $\left\{\beta_{1}, \ldots, \beta_{n-1}\right\}$ ) are real or appear in complex conjugate pairs; i.e., $\mathcal{L}_{n-1}=\mathcal{L}_{n-1}^{c}$ and $\dot{\circ}_{n-1}=\stackrel{\circ}{\mathcal{L}}_{n-1}^{c}$. Let $I_{n}(\stackrel{\circ}{F})=\sum_{k=1}^{n} \stackrel{\circ}{\lambda}_{k} \stackrel{\circ}{F}\left(z_{j}\right)$ be an nth rational Szegö quadrature formula with respect to $\stackrel{\circ}{\mu}$, given by (8), where the nodes $\left\{z_{k}=e^{\mathbf{i} \theta_{k}}\right\}_{k=1}^{n}$ are the zeros of the pORF $\stackrel{\circ}{Q}, \tau_{n}(z)=\phi_{n}(z)+\tau \phi_{n}^{*}(z)$ with $\tau \neq \pm 1$. Set $x_{k}=\cos \theta_{k}$, $k=1, \ldots, n$ and $\lambda_{k}=\AA_{k} / 2>0$, and consider the n-point rational interpolatory quadrature formula based upon these nodes and weights:

$$
J_{n}^{\tau}(F)=\sum_{k=1}^{n} \lambda_{k} F\left(x_{k}\right)
$$

for $J_{\mu}(F)=\int_{-1}^{1} F(x) d \mu(x)$. Then this rational interpolatory quadrature formula is exact for every $F \in \mathcal{L}_{n-1}$. Furthermore, it is exact in $\mathcal{L}_{n}$ iff

$$
\tau=-\phi_{n}\left(\beta_{n}\right) \pm \mathbf{i} \sqrt{1-\phi_{n}^{2}\left(\beta_{n}\right)} .
$$

Proof. Since for every function $F \in \mathcal{L}_{n-1}$ it holds that the corresponding function $\stackrel{\circ}{F} \in \stackrel{\circ}{\mathcal{L}}_{n-1}^{c}$. $\stackrel{\circ}{\mathcal{L}}_{(n-1) *}=\dot{\mathcal{L}}_{n-1} \cdot \stackrel{\circ}{(}(n-1) *$, it follows that

$$
J_{n}^{\tau}(F)=\frac{1}{2} I_{n}(\stackrel{\circ}{F})=\frac{1}{2} I_{\dot{\mu}}(\stackrel{\circ}{F})=J_{\mu}(F),
$$

implying that $J_{n}^{\tau}(F)$ is of interpolatory type. Next, let $F_{n}(x)=\frac{\prod_{k=1}^{n}\left(x-x_{k}\right)}{\pi_{n}(x)} \in \mathcal{L}_{n} \backslash \mathcal{L}_{n-1}$, then it holds that $J_{n}^{\tau}\left(F_{n}\right)=0$. So, let us see now for which set of nodes $\left\{x_{k}\right\}_{k=1}^{n}$ (or equivalently, for which values 
of $\tau$ ) it holds that $J_{\mu}\left(F_{n}\right)=0$. Consider the corresponding function $\stackrel{\circ}{F}_{n} \in \stackrel{\circ}{\mathcal{L}}_{n} \cdot \stackrel{\circ}{\mathcal{L}}_{n *}$, then it is easily verified that it is of the form

$$
\stackrel{\circ}{F}_{n}(z)=c_{n} \frac{\prod_{k=1}^{n}\left(z-z_{k}\right)\left(z-\bar{z}_{k}\right)}{\stackrel{\circ}{\pi}_{n}(z) \dot{\circ}_{n}^{*}(z)}, c_{n} \in \mathbb{C}_{0} .
$$

On the other hand we have that $\stackrel{\circ}{Q}, \tau \in \stackrel{\circ}{\mathcal{L}}_{n}$ is of the form

$$
\stackrel{\circ}{Q}_{n, \tau}(z)=d_{n} \frac{\prod_{k=1}^{n}\left(z-z_{k}\right)}{\stackrel{\circ}{\pi}_{n}(z)}, d_{n} \in \mathbb{C}_{0}
$$

so that

$$
B_{n *}(z) \stackrel{Q}{Q}_{n, \tau}^{c}(z)=\bar{v}_{n} \frac{\stackrel{\circ}{n}_{n}(z)}{\tilde{\pi}_{n}^{*}(z)} \cdot \bar{d}_{n} \frac{\prod_{k=1}^{n}\left(z-\bar{z}_{k}\right)}{\dot{\circ}_{n}(z)}=\bar{v}_{n} \bar{d}_{n} \frac{\prod_{k=1}^{n}\left(z-\bar{z}_{k}\right)}{\dot{\pi}_{n}^{*}(z)} .
$$

Consequently, we have with $e_{n}=v_{n} c_{n} /\left|d_{n}\right|^{2} \in \mathbb{C}_{0}$ that

$$
\begin{aligned}
\stackrel{\circ}{F}_{n}(z) & =e_{n} B_{n *}(z) \stackrel{\circ}{Q}_{n, \tau}(z) \stackrel{\circ}{Q}_{n, \tau}^{c}(z) \\
& =e_{n} B_{n *}(z)\left\{\phi_{n}(z)+\tau \phi_{n}^{*}(z)\right\}\left\{\phi_{n}(z)+\bar{\tau} \phi_{n}^{*}(z)\right\} \\
& =\hat{e}_{n}\left\{\phi_{n}(z)+\tau \phi_{n}^{*}(z)\right\}\left\{\phi_{n *}(z)+\tau \phi_{n *}^{*}(z)\right\}, \hat{e}_{n}=e_{n} \bar{\tau} .
\end{aligned}
$$

As a result,

$$
\begin{aligned}
J_{\mu}\left(F_{n}\right) & =\frac{\hat{e}_{n}}{2}\left[I_{\dot{\mu}}\left(\phi_{n} \phi_{n *}\right)+2 \mathfrak{R}\left\{I_{\dot{\mu}}\left(\phi_{n} \phi_{n *}^{*}\right)\right\} \tau+I_{\dot{\mu}}\left(\phi_{n}^{*} \phi_{n *}^{*}\right) \tau^{2}\right] \\
& =\frac{\hat{e}_{n}}{2}\left\|\phi_{n}\right\|_{\dot{\mu}}^{2}\left(1+2 \mathfrak{R}\left\{a_{n}\right\} \tau+\tau^{2}\right),
\end{aligned}
$$

where $\left\|\phi_{n}\right\|_{\mu}^{2}=\left\langle\phi_{n}, \phi_{n}\right\rangle_{\mu}>0$, and $\bar{a}_{n}$ represents the leading coefficient in the expansion of $\phi_{n}^{*}$ in the basis $\left\{B_{0}, \ldots, B_{n}\right\}$. Note that

$$
a_{n}=\left(\phi_{n}^{*}\right)^{*}\left(\beta_{n}\right)=\phi_{n}\left(\beta_{n}\right)=\phi_{n}^{c}\left(\beta_{n}\right)=\bar{a}_{n},
$$

and hence, is real. Furthermore, from [6, Cor. 3.1.4] we deduce that $1=\left|\phi_{n}^{*}\left(\beta_{n}\right)\right|^{2}>\left|\phi_{n}\left(\beta_{n}\right)\right|^{2}$, so that $J_{\mu}\left(F_{n}\right)=0$ iff $1+2 \phi_{n}\left(\beta_{n}\right) \tau+\tau^{2}=0$, yielding $\tau=-\phi_{n}\left(\beta_{n}\right) \pm \mathbf{i} \sqrt{1-\phi_{n}^{2}\left(\beta_{n}\right)}$.

Remark 3.10. In the previous theorem, we assumed that the poles were real or appeared in complex conjugate pairs. Therefore, we can consider the auxiliary subspaces $\tilde{\mathcal{L}}_{q} \subseteq \tilde{\mathcal{L}}_{p} \subseteq \mathcal{L}_{n}$, with $\tilde{\alpha}_{p} \in \overline{\mathbb{R}}_{I}$, so that $\mathcal{L}_{n}=\tilde{\mathcal{L}}_{p} \cdot \tilde{\mathcal{L}}_{q}^{c}=\tilde{\mathcal{R}}_{p, q}$ and $p-q$ is minimal (clearly, this difference $p-q$ then equals the number of real poles with odd multiplicity). Consequently, for $\tau \in\{ \pm 1\}$ the $n$-point rational interpolatory quadrature formula, given by (13), reduces to a rational Gauss-type quadrature formula based on an interpolating rational function in $\tilde{\mathcal{L}}_{p-1}$ (or, in $\tilde{\mathcal{L}}_{p}$ if $n$ is even and $\tau=-v_{n}$ ) whenever $p=\lceil n / 2\rceil$ and $q=\lfloor n / 2\rfloor$. When $p-q>1$, however, the $n$-point rational Szegó quadrature formula for $\tau \in\{ \pm 1\}$ cannot be related to one of the classical rational interpolating rules to obtain a quadrature formula for $J_{\mu}(F)=\int_{-1}^{1} F(x) d \mu(x)$. 


\section{Numerical examples}

In this section we will illustrate the results from the previous section by means of some numerical experiments. For this, we consider the Chebyshev weight function of the first kind $d \mu(x)=d x / \sqrt{1-x^{2}}$ on $I$, for which the corresponding measure $\stackrel{\circ}{\mu}$ on $\mathbb{T}$ is the Lebesgue measure $d \mu(z)=d z / \mathbf{i} z$. Our purpose then is to obtain a characterization of rational Gaussian, Gauss-Radau and Gauss-Lobatto rules associated with $\mu$ by giving explicit expressions for the corresponding nodal rational functions and weights in the quadrature formulas.

Given the sequence of complex numbers $\mathcal{B}_{n}=\left\{\beta_{1}, \beta_{2}, \ldots, \beta_{n}\right\} \subset \mathbb{D}$, the monic ORF with respect to the Lebesgue measure $\mu$ is the so-called Takenaka-Malmquist basis (see [16] and [20]), given by

$$
\phi_{n}(z)=\eta_{n}\left(1-\left|\beta_{n}\right|^{2}\right) \frac{z B_{n-1}(z)}{1-\bar{\beta}_{n} z}, \quad \text { and hence } \quad \phi_{n}^{*}(z)=\frac{1-\left|\beta_{n}\right|^{2}}{1-\bar{\beta}_{n} z},
$$

where $\eta_{n}$ is defined as before in (3). Further, the nodes $\left\{z_{k}\right\}_{k=1}^{n}$ in an $n$-point rational Szegó quadrature formula with respect to the Lebesgue measure $\stackrel{\circ}{\mu}$, based on the pORF ${\stackrel{\circ}{Q}, \tau_{n}}(z)=\phi_{n}(z)+\tau_{n} \phi_{n}^{*}(z)$, satisfy (see also [2, Sect. 4])

$$
z_{k} \eta_{n} B_{n-1}\left(z_{k}\right)=-\tau_{n},
$$

while the corresponding weights are given by (see also [6, Thm. 5.4.2])

$$
\stackrel{\circ}{k}_{k}=2 \pi\left\{1+\sum_{j=1}^{n-1} P\left(z_{k}, \beta_{j}\right)\right\}^{-1},
$$

where $P(z, \beta)$ denotes the Poisson kernel: $P(z, \beta)=\frac{1-|\beta|^{2}}{|z-\beta|^{2}}$, with $z \in \mathbb{T}$ and $\beta \in \mathbb{D}$.

Recall that for the rational Gaussian, Gauss-Radau and Gauss-Lobatto quadrature formulas we work with the sequence $\hat{\mathcal{B}}_{2 n}=\left\{\hat{\beta}_{1}, \ldots, \hat{\beta}_{2 n}\right\} \subset \mathbb{D}$ given by (7), and with $\beta_{n} \in(-1,1)$, so that

$$
\hat{B}_{2 k-1}(z)=B_{k}^{c}(z) B_{k-1}(z) \quad \text { and } \quad \hat{B}_{2 k}(z)=B_{k}(z) B_{k}^{c}(z) .
$$

Consequently, (9) along with (14)-(15) gives us the following expression for an $n$th ORF with respect to the measure $\mu$ on $I$ (see also [12, 17] and [9, Chapt. 3]):

$$
\varphi_{n}(x)=C_{n} \eta_{n}\left(1-\beta_{n}^{2}\right)\left\{\frac{z B_{n-1}^{c}(z)}{1-\beta_{n} z}+\frac{B_{(n-1) *}(z)}{z-\beta_{n}}\right\}, \quad C_{n} \in \mathbb{C}_{0} .
$$

Thus, the nodes $x_{k}=J\left(z_{k}\right)$ of the $n$-point rational Gaussian quadrature formula $J_{n}(F)$ satisfy

$$
z_{k}\left(\frac{z_{k}-\beta_{n}}{1-\beta_{n} z_{k}}\right) B_{n-1}\left(z_{k}\right) B_{n-1}^{c}\left(z_{k}\right)=-1
$$

while the weights are given by

$$
\lambda_{k}=2 \pi\left\{1+\sum_{j=1}^{n-1}\left[P\left(z_{k}, \beta_{j}\right)+P\left(z_{k}, \bar{\beta}_{j}\right)\right]+P\left(z_{k}, \beta_{n}\right)\right\}^{-1} .
$$


Next, in a similar way we deduce from (10) and (14)-(15) that

$$
\tilde{\varphi}_{n-1}(x)=C_{n-1}\left\{\frac{z\left(z-\beta_{n}\right) B_{n-1}^{c}(z)-\left(1-\beta_{n} z\right) B_{(n-1) *}(z)}{z^{2}-1}\right\}, \quad C_{n-1} \in \mathbb{C}_{0} .
$$

Thus, the nodes $x_{k}=J\left(z_{k}\right)$ in the $(n+1)$-point rational Gauss-Lobatto formula $J_{n+1}(F)$ with fixed nodes in $x=1$ and $x=-1$ satisfy

$$
z_{k}\left(\frac{z_{k}-\beta_{n}}{1-\beta_{n} z_{k}}\right) B_{n-1}\left(z_{k}\right) B_{n-1}^{c}\left(z_{k}\right)=1,
$$

whereas the weights are now given by (see Theorem 3.4)

$$
\lambda_{k}=\left\{\begin{array}{cc}
2 \pi\left\{1+\sum_{j=1}^{n-1}\left[P\left(z_{k}, \beta_{j}\right)+P\left(z_{k}, \bar{\beta}_{j}\right)\right]+P\left(z_{k}, \beta_{n}\right)\right\}^{-1}, & z_{k}^{2} \neq 1 \\
\pi\left\{1+\sum_{j=1}^{n-1}\left[P\left(z_{k}, \beta_{j}\right)+P\left(z_{k}, \bar{\beta}_{j}\right)\right]+P\left(z_{k}, \beta_{n}\right)\right\}^{-1}, & z_{k}^{2}=1 .
\end{array}\right.
$$

Finally, after some computations (11) and (14)-(15) yield that

$$
\breve{\varphi}_{n-1}^{ \pm}(x)=\frac{\eta_{n}\left(1-\beta_{n}^{2}\right)}{(z \pm 1)\left( \pm 1-\beta_{n}\right)}\left\{z B_{n-1}^{c}(z) \pm B_{(n-1) *}(z)\right\} .
$$

So, the nodes $x_{k}^{ \pm}=J\left(z_{k}^{ \pm}\right)$in the $n$-point rational Gauss-Radau formula $J_{n}(F)$ with fixed node in $x=\mp 1$ satisfy

$$
z_{k}^{ \pm} B_{n-1}\left(z_{k}^{ \pm}\right) B_{n-1}^{c}\left(z_{k}^{ \pm}\right)=\mp 1
$$

and the weights are given by (see Theorem 3.7)

$$
\lambda_{k}^{ \pm}= \begin{cases}2 \pi\left\{1+\sum_{j=1}^{n-1}\left[P\left(z_{k}^{ \pm}, \beta_{j}\right)+P\left(z_{k}^{ \pm}, \bar{\beta}_{j}\right)\right]\right\}^{-1}, & z_{k}^{ \pm} \neq \mp 1 \\ \pi\left\{1+\sum_{j=1}^{n-1}\left[P\left(z_{k}, \beta_{j}\right)+P\left(z_{k}, \bar{\beta}_{j}\right)\right]\right\}^{-1}, & z_{k}^{ \pm}=\mp 1 .\end{cases}
$$

In the numerical examples that follow, we used the above expressions to compute the nodes and weights in the rational quadrature rule on $I$. In [24] a numerical procedure is described to compute the nodes and weights in the rational Gaussian quadrature rule up to machine precision in $O(m \cdot n)$ flops, where $n$ denotes the number of interpolation points and $m$ represents the number of different poles (two poles $\alpha_{j}$ and $\alpha_{k}$ were assumed to be different if $\alpha_{j} \neq \alpha_{k}$ and $\alpha_{j} \neq \bar{\alpha}_{k}$ ). This numerical procedure was based on the above expressions for the rational Gaussian nodes and weights, and was implemented in MATLAB ${ }^{\circledR 11}$. A similar procedure can be used to compute the nodes and weights in the rational Gauss-Radau and Gauss-Lobatto quadrature rules. However, due to time restrictions we decided to use a simple (short) and straightforward, but less efficient (and probably also less accurate for large $n$ ) implementation in MAPLE ${ }^{\circledR 12}$ instead. All the computations were done with 30 digits in MAPLE ${ }^{\circledR} 9.5$.

\footnotetext{
${ }^{11}$ MATLAB is a registered trademark of The MathWorks, Inc.

${ }^{12}$ MAPLE is a registered trademark of Waterloo Maple, Inc.
} 
Example 4.1. Define the function $f^{[\alpha]}(x)=\frac{\alpha x-1}{x-\alpha}$, with $\alpha=J(\beta) \in \overline{\mathbb{C}}_{I}$. In [22, Thm. 3.2(2)] it is proved that

$$
J_{\mu}\left(f^{[\alpha]}\right)=\int_{-1}^{1} f^{[\alpha]}(x) \frac{d x}{\sqrt{1-x^{2}}}=\pi \beta .
$$

Consider now the sequence of poles $\left\{\alpha_{1}, \ldots, \alpha_{4}\right\}=\left\{-\frac{3}{4} \mathbf{i}, \frac{5}{4}, \frac{3-\mathbf{i}}{4}, \infty\right\}$. We then numerically compute $J_{\mu}\left(f_{1}\right)$ and $J_{\mu}\left(f_{2}\right)$, with

$$
f_{1}(x)=\frac{1}{\pi} \mathfrak{R}\left\{f^{\left[\alpha_{1}\right]}(x)+f^{\left[\alpha_{2}\right]}(x)+f^{\left[\alpha_{3}\right]}(x)\right\} \quad \text { and } \quad f_{2}(x)=f_{1}(x)+\frac{x}{\pi},
$$

by means of the $n$-point rational Gaussian (respectively $(n+1)$-point rational Gauss-Lobatto and $n$-point rational Gauss-Radau) quadrature formula, based on the sequence of poles

$$
\left\{\alpha_{1}, \ldots, \alpha_{n-1}, \infty\right\}, \quad 2 \leqslant n \leqslant 5 .
$$

Note that the exact solution is $J_{\mu}\left(f_{1}\right)=J_{\mu}\left(f_{2}\right)=1$. Next, we approximate $J_{\mu}\left(f_{1}\right)$ and $J_{\mu}\left(f_{2}\right)$ by means of an $n$-point rational interpolatory quadrature formula, based on the sequence of poles

$$
\left\{\alpha_{1}, \bar{\alpha}_{1}, \ldots, \alpha_{k}, \bar{\alpha}_{k}, \infty, \infty\right\} \text { if } n=2 k+2 \text {, or }\left\{\alpha_{1}, \bar{\alpha}_{1}, \ldots, \alpha_{k}, \bar{\alpha}_{k}, \infty\right\} \text { if } n=2 k+1,
$$

for $4 \leqslant n \leqslant 8$, with $\tau_{n}=e^{\mathbf{i} \frac{4 \pi}{3}}=-\frac{1}{2}(1+\sqrt{3} \mathbf{i})$ and with $\tau_{n}=-\mathbf{i}$ (i.e., the optimal value for $\left.\tau_{n} \in \mathbb{T} \backslash\{ \pm 1\}\right)$. Tables 1-3 then show the relative error on the approximation: Error $i=$ $\left|J_{n}\left(f_{i}\right)-J_{\mu}\left(f_{i}\right)\right| /\left|J_{\mu}\left(f_{i}\right)\right|, i=1,2$. These tables clearly show that the approximation is exact whenever the integrand $f_{i}$ is in the domain of validity.

\begin{tabular}{|c|c|c|}
\hline$n$ & Error 1 & Error 2 \\
\hline 2 & $4.07894 E-01$ & $4.07894 E-01$ \\
\hline 3 & $5.73566 E-02$ & $5.73566 E-02$ \\
\hline 4 & 0 & 0 \\
\hline 5 & 0 & 0 \\
\hline
\end{tabular}

\begin{tabular}{|c|c|c|}
\hline$n$ & Error 1 & Error 2 \\
\hline 2 & $2.50000 E-01$ & $2.50000 E-01$ \\
\hline 3 & $7.39299 E-02$ & $7.39299 E-02$ \\
\hline 4 & $1.00000 E-29$ & 0 \\
\hline 5 & 0 & 0 \\
\hline
\end{tabular}

Table 1: Relative errors in the $n$-point rational Gaussian (left) and $(n+1)$-point rational Gauss-Lobatto (right) quadrature formulas for the estimation of $J_{\mu}\left(f_{i}\right)$, where $f_{i}$ is given by (16) for $i=1,2$.

\begin{tabular}{|c|c|c|}
\hline$n$ & Error 1 & Error 2 \\
\hline 2 & $9.01098 E-01$ & $6.51098 E-01$ \\
\hline 3 & $6.84596 E-02$ & $1.30959 E-01$ \\
\hline 4 & $1.00000 E-29$ & $3.12499 E-02$ \\
\hline 5 & 0 & 0 \\
\hline
\end{tabular}

\begin{tabular}{|c|c|c|}
\hline$n$ & Error 1 & Error 2 \\
\hline 2 & $8.14479 E-01$ & $5.64479 E-01$ \\
\hline 3 & $1.40077 E-01$ & $2.02577 E-01$ \\
\hline 4 & $1.00000 E-29$ & $3.12500 E-02$ \\
\hline 5 & $4.00000 E-30$ & 0 \\
\hline
\end{tabular}

Table 2: Relative errors in the $n$-point rational Gaussian-Radau rules, with a fixed node in $x=-1$ (left) or $x=1$ (right), for the estimation of $J_{\mu}\left(f_{i}\right)$, where $f_{i}$ is given by (16) for $i=1,2$. 


\begin{tabular}{|c|c|c|}
\hline$n$ & Error 1 & Error 2 \\
\hline 4 & $1.50199 E-01$ & $1.50199 E-01$ \\
\hline 5 & $2.54437 E-02$ & $5.66937 E-02$ \\
\hline 6 & $2.71619 E-02$ & $2.71619 E-02$ \\
\hline 7 & $1.00000 E-29$ & $1.56250 E-02$ \\
\hline 8 & 0 & $1.00000 E-29$ \\
\hline
\end{tabular}

\begin{tabular}{|c|c|c|}
\hline$n$ & Error 1 & Error 2 \\
\hline 4 & $6.28555 E-02$ & $6.28555 E-02$ \\
\hline 5 & $3.48186 E-02$ & $3.48186 E-02$ \\
\hline 6 & $8.03880 E-03$ & $8.03880 E-03$ \\
\hline 7 & $6.00000 E-30$ & $1.00000 E-30$ \\
\hline 8 & $2.00000 E-30$ & 0 \\
\hline
\end{tabular}

Table 3: Relative errors in the $n$-point rational interpolatory quadrature formulas, with $\tau_{n}=e^{\mathbf{i} \frac{4 \pi}{3}}($ left $)$ and $\tau_{n}=-\mathbf{i}$ (right), for the estimation of $J_{\mu}\left(f_{i}\right)$, where $f_{i}$ is given by (16) for $i=1,2$.

Example 4.2. Finally, consider the function

$$
f_{3}(x)=\frac{1}{\pi} \sin \left(\frac{1}{x^{2}-\omega^{2}}\right), \quad \omega \in \mathbb{R}_{I},
$$

which is taken from [23, Ex. 5.7]. This function has an essential singularity in $x=\omega$ and $x=-\omega$. For $\omega>1$ but very close to 1 , this function is extremely oscillatory near these singularities. Since an essential singularity can be viewed as a pole of infinity multiplicity, this suggests taking

$$
\alpha_{k}=(-1)^{k} \omega, k=1, \ldots, n .
$$

For $\omega=25 / 24$ we obtain with the aid of MAPLE ${ }^{\circledR} 9.5$ that $J_{\mu}\left(f_{3}\right) \approx-0.458645566330016385598360747362$. First, we approximate $J_{\mu}\left(f_{3}\right)$ by means of the $n$-point rational Gaussian (respectively $(n+1)$-point rational Gauss-Lobatto and $n$-point rational Gauss-Radau) quadrature formula, based on the sequence of poles (18), for $n=3, \ldots, 15$. Figure 1 then shows the relative error $\left|J_{n}\left(f_{3}\right)-J_{\mu}\left(f_{3}\right)\right| /\left|J_{\mu}\left(f_{3}\right)\right|$ as a function of the number of nodes in the quadrature formula (fixed nodes included). For the case of rational Gauss-Radau, we only plotted the results for a fixed node in $x=1$ (the results for a fixed node in $x=-1$ are practically the same). Next, we approximate $J_{\mu}\left(f_{3}\right)$ by means of an $n$-point rational interpolatory quadrature formula, based on the same sequence of poles, with $\tau_{n}=e^{\mathbf{i} \frac{4 \pi}{3}}=-\frac{1}{2}(1+\sqrt{3} \mathbf{i})$ and with $\tau_{n}=-\mathbf{i}$ (i.e., the optimal value for $\left.\tau_{n} \in \mathbb{T} \backslash\{ \pm 1\}\right)$. Figure 2 then shows the relative error on the approximation, together with the relative error when the classical Gaussian quadrature formula (i.e., all the poles $\alpha_{k}$ at infinity) is used.

Note that we already obtain 18 correct digits with the 15-point rational Gauss-Radau quadrature formula, while the classical Gaussian quadrature formula barely provides us with one correct digit for the same number of nodes. To obtain the same accuracy of 18 correct digits using the classical Gaussian quadrature formula we needed $n=112$ nodes.

Finally, for $n=2 m+1, m=1,2, \ldots$, it holds that the interpolating rational functions have poles in both the singularities of $f_{3}$, each with the same multiplicity $m$. This may explain the oscillating behavior of the relative errors as a function of $n$, with better results whenever $n$ is odd for most of the rational quadrature rules. However, it does not explain why the opposite (i.e.; better results whenever $n$ is even) occurs for the rational Gaussian quadrature rule. Further, we have that the rational Gauss-Radau quadrature rule has domain of validity $\mathcal{R}_{n-1, n-1}=\mathcal{L}\left\{(-1)^{j} \omega, j=\right.$ $1, \ldots, 2(n-1)\}$. Thus, both the singularities of $f_{3}$ have the same multiplicity $n-1$ in the sequence 


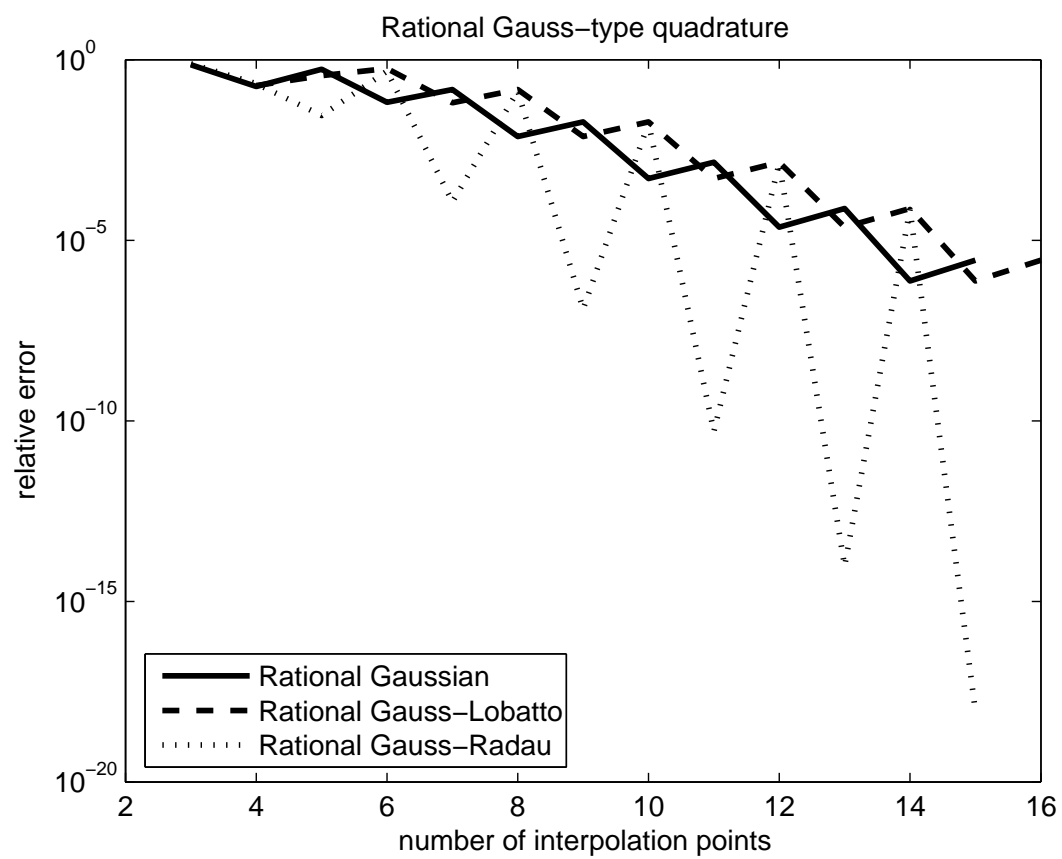

Figure 1: Relative error in the rational Gauss-type quadrature formulas for the estimation of $J_{\mu}\left(f_{3}\right)$, where $f_{3}$ is given by (17).

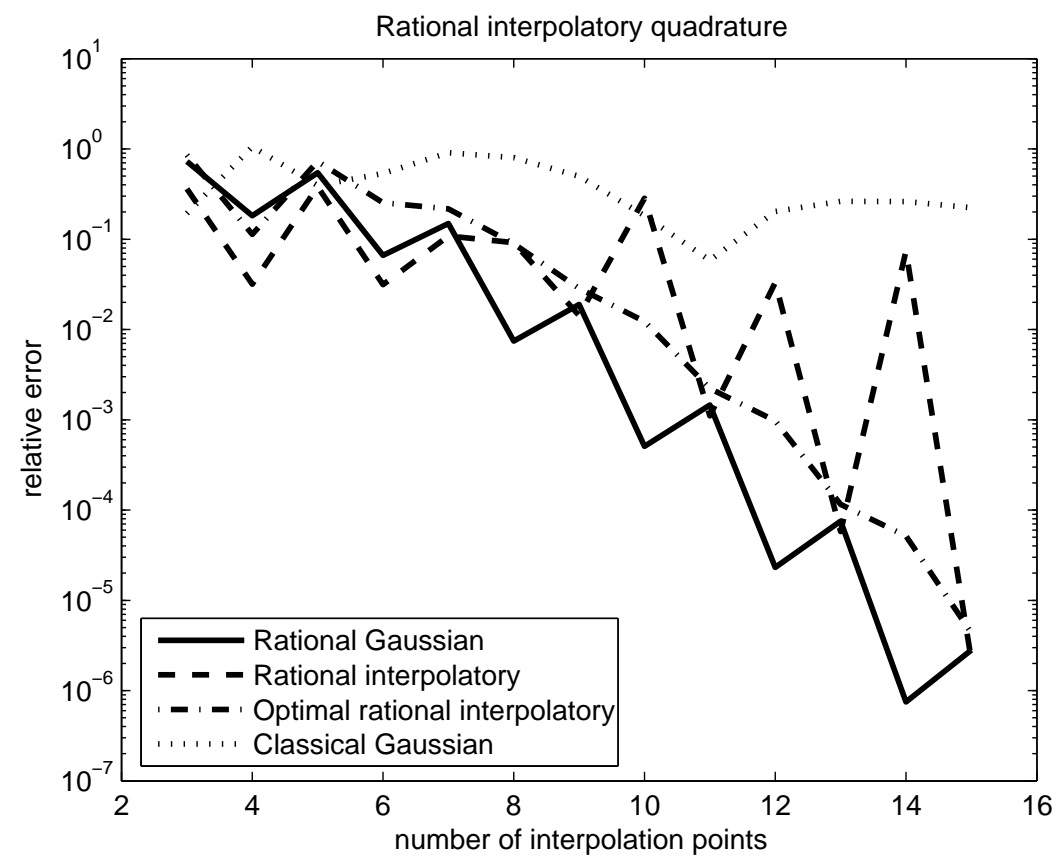

Figure 2: Relative error in the rational interpolatory quadrature formulas, with $\tau_{n}=e^{\mathbf{i} \frac{\mathbf{4}}{3}}=-\frac{1}{2}(1+\sqrt{3} \mathbf{i})$ and $\tau_{n}=-\mathbf{i}$, and in the rational and classical Gaussian quadrature formulas for the estimation of $J_{\mu}\left(f_{3}\right)$, where $f_{3}$ is given by (17). 
of poles corresponding to the space $\mathcal{R}_{n-1, n-1}$, which may explain the better results for rational Gauss-Radau (compared with the rational Gaussian quadrature rule) whenever $n$ is odd.

\section{Acknowledgements}

Most of this work was done during a research stay of the first author at the Department of Mathematical Analysis of La Laguna University, Tenerife (Spain). This author is very grateful to Professor Pablo González-Vera, dr. Ruymán Cruz-Barroso, and Francisco Perdomo-Pío, for their hospitality at the La Laguna University, and for the valuable discussions and comments during the development of this work.

\section{References}

[1] W. Blaschke, Erweiterung des Satzes von Vitali über Folgen analytischer Funktionen, Berichte über die Verhandlungen der Königlich-Sächsische Gesellschaft der Wissenschaften zu Leipzig, Mathematisch-Physische Klasse 67 (1915) 194-200.

[2] A. Bultheel, R. Cruz-Barroso, K. Deckers, P. González-Vera, Rational szegő quadratures associated with chebyshev weight functions, Mathematics of Computation 78 (266) (2008) 1031-1059.

[3] A. Bultheel, R. Cruz-Barroso, M. Van Barel, On Gauss-type quadrature formulas with prescribed nodes anywhere on the real line, Calcolo 47 (1) (2010) 28-41.

[4] A. Bultheel, L. Daruis, P. González-Vera, A connection between quadrature formulas on the unit circle and the interval [-1, 1], Journal of Computational and Applied Mathematics 132 (1) (2001) 1-14.

[5] A. Bultheel, L. Daruis, P. González-Vera, Positive interpolatory quadrature formulas and para-orthogonal polynomials, Journal of Computational and Applied Mathematics 179 (1-2) (2005) 97-119.

[6] A. Bultheel, P. González-Vera, E. Hendriksen, O. Njåstad, Orthogonal rational functions, vol. 5 of Cambridge Monographs on Applied and Computational Mathematics, Cambridge University Press, 1999.

[7] A. Bultheel, P. González-Vera, E. Hendriksen, O. Njåstad, Rational quadrature formulas on the unit circle with prescribed nodes and maximal domain of validity, IMA Journal Numererical Analysis. Published on line August 21, 2009.

[8] A. Bultheel, P. González-Vera, E. Hendriksen, O. Njåstad, Computation of rational Szegő-Lobatto quadrature formulas (Submitted).

[9] K. Deckers, Orthogonal rational functions: Quadrature, recurrence and rational Krylov, Ph.D. thesis, K.U.Leuven (February 2009).

[10] K. Deckers, A. Bultheel, J. Van Deun, A generalized eigenvalue problem for quasi-orthogonal rational functions (Submitted).

[11] K. Deckers, J. Van Deun, A. Bultheel, An extended relation between orthogonal rational functions on the unit circle and the interval [-1, 1], Journal of Mathematical Analysis and Applications 334 (2) (2007) 1260-1275.

[12] K. Deckers, J. Van Deun, A. Bultheel, Rational Gauss-Chebyshev quadrature formulas for complex poles outside $[-1,1]$, Mathematics of Computation 77 (262) (2008) 967-983.

[13] K. Deckers, J. Van Deun, A. Bultheel, Computing rational gauss-chebyshev quadrature formulas with complex poles: the algorithm, Advances in Engineering Software 40 (8) (2009) 707-717.

[14] G. Freud, Orthogonal polynomials, Pergamon Press, Oxford, 1971.

[15] C. Jagels, L. Reichel, Szegő-Lobatto quadrature rules, Journal of Computational and Applied Mathematics 200 (1) (2007) 116-126.

[16] F. Malmquist, Sur la détermination d'une classe de fonctions analytiques par leurs valeurs dans un ensemble donné de points, C.R. 6ième Congrès des Math. Scand., Gjellerups, Copenhagen (1926) 253-259.

[17] E. A. Rovba, Orthogonal systems of rational functions on the segment and quadratures of gauss-type, Mathematica Balkanica (N.S.) 13 (1-2) (1999) 187-198.

[18] W. Rudin, Real and complex analysis, 3rd ed., McGraw-Hill, New York, 1987. 
[19] G. Szegő, Orthogonal polynomials, vol. 33 of Amer. Math. Soc. Colloq. Publ., 4th ed., American Mathematical Society, Providence, Rhode Island, 1975, first edition 1939.

[20] S. Takenaka, On the orthogonal functions and a new formula of interpolation, Japanese Journal of Mathematics 2 (1925) 129-145.

[21] J. Van Deun, A. Bultheel, Orthogonal rational functions and quadrature on an interval, Journal of Computational and Applied Mathematics 153 (1-2) (2003) 487-495.

[22] J. Van Deun, A. Bultheel, A quadrature formula based on chebyshev rational functions, IMA Journal of Numerical Analysis 26 (4) (2006) 641-656.

[23] J. Van Deun, A. Bultheel, P. González-Vera, On computing rational Gauss-Chebyshev quadrature formulas, Mathematics of Computation 75 (253) (2006) 307-326.

[24] J. Van Deun, K. Deckers, A. Bultheel, J. A. C. Weideman, Algorithm 882: Near best fixed pole rational interpolation with applications in spectral methods, ACM Transactions on Mathematical Software 35 (2) (2008) $14: 1-14: 21$.

[25] P. Van gucht, A. Bultheel, A relation between orthogonal rational functions on the unit circle and the interval $[-1,1]$, Communications in the Analytic Theory of Continued Fractions 8 (2000) 170-182. 